\title{
Error sensitivity analysis of machine tools based on multi-body kinematic theory
}

\author{
Jinwei Fan ${ }^{1, a}$, Shuai Yuan ${ }^{2, b}$ and Lanqing Zhang ${ }^{2, c}$ \\ ${ }^{1}$ School of Mechanical Engineering, Beijing University of Technology, Beijing100124, China; \\ ${ }^{2}$ School of Mechanical Engineering, Beijing University of Technology, Beijing100124, China. \\ ajwfan@bjut.edu.cn, b530211126@qq.com, 'c1002878319@qq.com
}

Keywords: multi-body kinematics; sensitivity; error modeling; geometric error; error parameters

\begin{abstract}
In order to determine the key factors which have an impact on the machining accuracy of a machine tool after its machining errors occur, a different method analyzing the sensitivity of machine tool errors based on the theory of multi-body system kinematics is proposed. Firstly, the method assumes a machine tool to be several rigid bodies according to the theory of multi-body system kinematics, establishes the body reference coordinate system and the kinematic reference coordinate system on each rigid body, and constructs the constraint equation by using the method of describing the center of the tool and the machining point in the system coordinates to solve the machining errors of the machine tool. Then, it gives the sensitivities of error parameters by taking the derivative of errors and establishes the impact analysis model. The calculation and analysis for an example show that when using the method the fundamental factors that have significant influence on machine tool errors can be identified efficiently. It can provide the theoretical basis for designers and users to improve machine tools.
\end{abstract}

\section{Introduction}

With the continuous development of the manufacturing industry, CNC machine tools as manufacturing industry machine tools and its function in the continuous improvement, application scope expanding, accordingly, the requirements on machining accuracy of NC machine tool also continues to increase, the machining accuracy of NC machine tool has become measure a country's level of science and technology is one of the important standards. People all know, manufacturing errors of CNC machine tools can reflect the level of the actual accuracy of CNC machine tools, machining error is the ultimate reflection of $\mathrm{CNC}$ machine tools to all parts of the coupling error function, how to according to the machining error to error traceability, find the cause machining error is the main factor, is currently plaguing the machine tool designer and use Therefore, the use of reasonable error analysis model, to determine the accuracy of the error parameters, the error sensitivity analysis of machine tools is of great significance.

There are numerous factors that affect the machining accuracy of machine tools, the main geometric error, thermal error, servo system error and load error, thermal deformation and force deformation are expressed by means of geometric error. Therefore, the geometric error parameters can be written in the form of geometric error parameters. So the error parameters can be utilized to express. The relationship between the influence of the sensitivity of the position error of the differential applications due to system analysis and other methods. At present, many body theory of complex system is highly generalization and abstraction, fully consider the influence of various factors and their coupling relationship, which have been widely applied to the machine tool error modeling and analysis. Yang Qiang in-depth analysis of a novel parallel mechanism of the principal design variables to determine the reasonable design parameters. Qiang and other matrix differential method is used to deduce the platform position and orientation error and 42 kinds of original error, the definition of the platform position and orientation errors of the original error sensitivity is given according to the sensitivity of the proportion of the original error In this paper, the sensitivity analysis of NC machine tools is carried out, and the key factors of NC machine tools are studied. In this case, the sensitivity and influence the degree of the axis NC machine tools are analyzed. In this 
case, the sensitivity and influence the degree of the 5 axis NC machine tool are analyzed. Degree and impact parameters.

\section{Motion error modeling of multibody systems}

Geometric description method of adjacent body.Numerical control machine tool is composed of a number of rigid bodies, in the rigid body to establish their own reference frame, motion reference coordinate system, the machine tool can be used to coordinate the movement between the axis of each axis of motion between the coordinate system transformation, so that the machine tool accuracy analysis model including the full motion of the entire motion parameters.

Error modeling for five axis NC machine tool. The five axis NC machine tool with double head is flexible, the position of the tool can be arbitrarily adjusted, and the attitude of the cutter can be adjusted to the complex surface of the machining process.

Figure 1 shows the C-A type five axis NC machine, which has 3 moving axes and 2 rotational axes, and the 2 rotary axes are in the tool movement chain. The $Y$ move to the mobile and $Z$ to move, the A moves toward the rotary motion, and the $\mathrm{C}$ is a special kind of multi-body system. Therefore, the rigid body has six errors (3 lines. Displacement errors and three angular displacement errors), for five axis NC machine tools, a total of five body movement, a total of 30 errors. These both changes with the movement of the corresponding movement of the machine tool parts. In errors addition three translational axes between pairwise exist three non Perpendicularity Error, at the same time, turn a shaft and $\mathrm{Y}, \mathrm{Z}$ axis, the $\mathrm{C}$ and $\mathrm{X}, \mathrm{Y}$ axis exist four squareness errors. These errors are not random bed moving parts movement and change. Therefore the five axis machine tool is related to the error parameters of 37, as showed in Table 1 below.

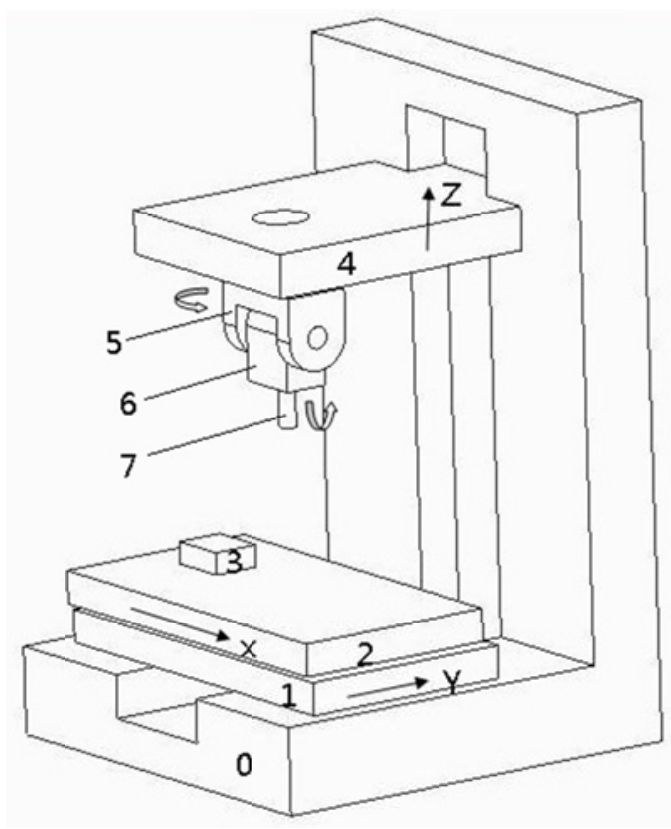

Figure 1 
Table 1

\begin{tabular}{|l|c|c|c|c|c|c|}
\hline $\begin{array}{l}\text { Error } \\
\text { property }\end{array}$ & \multicolumn{2}{|c|}{ Linear displacement error } & \multicolumn{3}{c|}{ Angular displacement error } \\
\hline $\begin{array}{l}\text { Error } \\
\text { direction }\end{array}$ & Along X & Along Y & Along Z & Along X & Along Y & Along Z \\
\hline $\begin{array}{l}\text { Translation } \\
\text { along X }\end{array}$ & $\delta_{x}(x)$ & $\delta_{y}(x)$ & $\delta_{z}(x)$ & $\varepsilon_{x}(x)$ & $\varepsilon_{y}(x)$ & $\varepsilon_{z}(x)$ \\
\hline $\begin{array}{l}\text { Translation } \\
\text { along Y }\end{array}$ & $\delta_{x}(y)$ & $\delta_{y}(y)$ & $\delta_{z}(y)$ & $\varepsilon_{x}(y)$ & $\varepsilon_{y}(y)$ & $\varepsilon_{z}(y)$ \\
\hline $\begin{array}{l}\text { Translation } \\
\text { along Z }\end{array}$ & $\delta_{x}(z)$ & $\delta_{y}(z)$ & $\delta_{z}(z)$ & $\varepsilon_{x}(z)$ & $\varepsilon_{y}(z)$ & $\varepsilon_{z}(z)$ \\
\hline Z rotation & $\delta_{x}(C)$ & $\delta_{y}(C)$ & $\delta_{z}(C)$ & $\varepsilon_{x}(C)$ & $\varepsilon_{y}(C)$ & $\varepsilon_{z}(C)$ \\
\hline X rotation & $\delta_{x}(A)$ & $\delta_{y}(A)$ & $\delta_{z}(A)$ & $\varepsilon_{x}(A)$ & $\varepsilon_{y}(A)$ & $\varepsilon_{z}(A)$ \\
\hline Verticality & $\varepsilon_{x y}, \varepsilon_{y z}, \varepsilon_{x z}, \varepsilon_{A y}, \varepsilon_{A z}, \varepsilon_{C x}, \varepsilon_{C y}$ \\
\hline
\end{tabular}

In order to describe these 37 errors, we should consider the feasibility of the detection method, and the coordinate system is set as follows: in the initial conditions, the reference frame of the moving parts is coincident with its reference frame. The coordinate system is related to the direction and position of the space.Firstly, determine the direction of each coordinate system: Select the direction of the machine tool coordinate system as the reference direction, make the bed (1) direction of the coordinate system and $\mathrm{Y}$ to the slide carriage ( 2 body) of the motion reference coordinate system and reference coordinate direction, make reference coordinate around its $\mathrm{Z}$ axis turn verticality $\varepsilon_{x y}$ coordinate system is workpiece coordinate system and Xdirection slide carriage ( 3 body) of motion reference coordinate system, make reference coordinate around its $\mathrm{X}$ 、 Yaxis turn verticality $^{\varepsilon_{y z}} 、 \varepsilon_{x z}$ coordinate system is workpiece coordinate system and Zdirection slide carriage ( 4 body) of motion reference coordinate system, make $Z$ direction slide carriage's motion reference coordinate system respectively around the $\mathrm{X}, \mathrm{Y}$ axis through verticality ${ }_{C X} 、{ }^{\varepsilon_{C y}}$ Coordinate system is Caxis ( 5 body) motion reference coordinate system, make $\mathrm{C}$ direction slide carriage's motion reference coordinate system respectively around the $\mathrm{Y} 、$ Zaxis through verticality ${ }^{\varepsilon_{A y}} 、 \varepsilon_{A z}$ Coordinate system is A axis (6 body) motion reference coordinate system. By the above method, we can determine the orientation of all the coordinates of the initial conditions.Determine the position of each coordinate system. The various moving parts of the machine tool are returned to the absolute zero of the numerical control machine tool,This initial conditions, bed set, $\mathrm{X}$ to slide, $\mathrm{y}$ to sneak in the reference coordinate system and the moving reference frame origin are located in the machine working table of a given point (O1points in Figure 2.). Body reference frame of $\mathrm{Z}$ direction slide carriage and $\mathrm{C}$ axis and its' motion reference coordinate system origin is located center of main shaft end (O4points in Figure 2), A axis and the reference frame of the reference frame of the tool frame and its' motion reference coordinate system origin is located at the center of the A axis rotation (O6points in Figure 2). According to the above method, the ideal characteristic matrix and the error characteristic matrix of five axis $\mathrm{NC}$ machine tool are obtained.Figure 3 shows the topology of the 3 axis $\mathrm{CNC}$ machine tool with a dual head,T body and $\mathrm{W}$ body respectively representthe tool of the A axis (6 body) and the workpiece of Xdirection slide carriage ( 3 body) . 


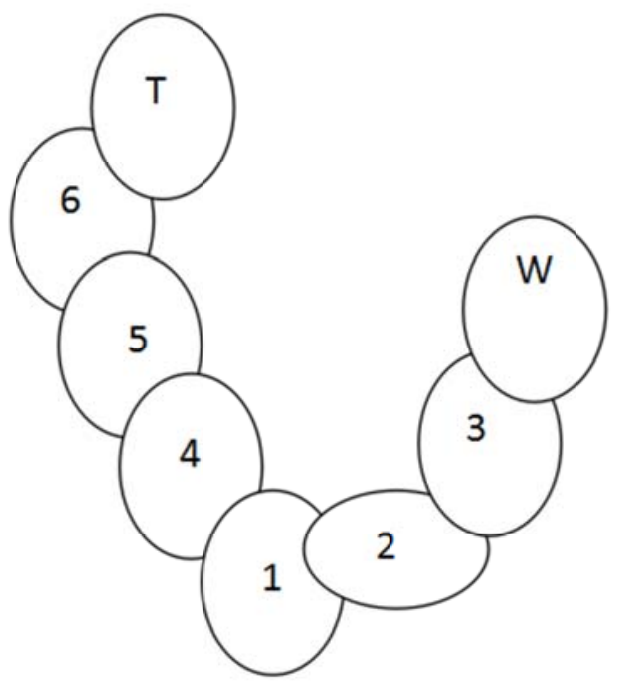

Figure 2

\section{Influence degree analysis of error parameters}

Different error parameters have different influence on machining accuracy of machine tools. Although there are some errors in the workpiece coordinate system, the error parameters are very small. Therefore, this paper puts forward the following model to evaluate the impact of the error parameters. The error parameter influences function can be expressed in the following form : ${ }^{\Delta=\frac{\partial E}{\partial u} u}$. $u$ is error parameter, $E$ is error.

\section{Summary}

Based on the theory of multi-body system kinematics, the reasonable error parameters established double pendulum head 5 axis $\mathrm{CNC}$ machine tool error analysis model, expression equation of motion error is obtained by using this model, through the way of error parameter one by one derivation, it is concluded that the equation of each error parameter sensitivity and error parameters. Through examples available angular displacement error sensitivity is relatively high. But overall, linear displacement of machine tool error influence is bigger. In the design of the machine should accord to the different conditions of use to determine the related key parameters and optimized.

\section{References}

[1] Pott A, Kecskemethy A, Hiller M. A simplified. force-based method for the linearization and sensitivity analysis of complex manipulation system. Mechanism and Machine Theory,2007,42:1445-1461.

[2] Habibi M, Arezoo B, Nojedeh M V. Tool deflection and geometrical error compensation by tool path modification. International Journal of Machine Tools \& Manufacture, 2011,51:439-449.

[3] Zhu S W, Ding G F, Qin S F, et al. Integrated geometric error modeling, identification and compensation of CNC machine tools. International Journal of Machine Tools \& Manufacture, 2012,52:24-49.

[4] Ibaraki S, Knapp W. Indirect measurement of volumetric accuracy for three-axis and five-axis machine tools: A Review. International Journal ofAutomationTechnology, 2012,6 :110-124.

[5] Khan A W , Chen W Y. A methodology for systematic geometric error compensation in five-axis machine tools. The International Journal of Advanced ManufacturingTechnology, $2011,53: 615-628$. 